\title{
Pi Sigma Alpha Names Award Winners
}

Pi Sigma Alpha, the National Political Science Honor Society, is pleased to announce its program award winners for 1998-99. Eleven students, four political science faculty members, and six local campus chapters of the honor society are being recognized for outstanding merit this year through the following awards.

\section{IIEA/Howard Penniman Scholarships for Graduate Study in Political \\ Science}

Awarded to members of the honor society who are entering graduate school in the fall of 1999. The winners receive $\$ 1500$ each.

\section{Gladys Annette Ganiel, Providence} College

Aaron Zachariah Hale, Texas A\&M University

Jennifer Lee Hildreth, University of Notre Dame

Neil G. Ruiz, University of California, Berkeley

Daniel Andrew Weiss, University of Pittsburgh

\section{Best Chapter Awards}

For local chapters that are extraordinarily active and effective within their departments, on their campuses, and in their communities. Prizes of $\$ 250$ are given in three categories based on the size of the institution in which the chapter is located.

\section{Schools with under 6,000 enrollment}

Upsilon Lambda Chapter, Saint Anselm's College. Dr. Paul C. Manuel, Advisor

Gamma Sigma Chapter, University of the South. Dr. Joan S. Ward, Advisor

\section{Schools with 6,000-15,000 enrollment}

Upsilon lota Chapter, Mercer University. Dr. Eimad C. Houry, Advisor

Delta Gamma Chapter, Northeastern University. Dr. Christopher J. Bosso, Advisor

\section{Schools with over 15,000 enrollment}

Beta Mu Chapter, Brigham Young University. Mr. Ray Christensen, Advisor

Delta Omega Chapter, Purdue University. Dr. Rosalee Clawson, Advisor

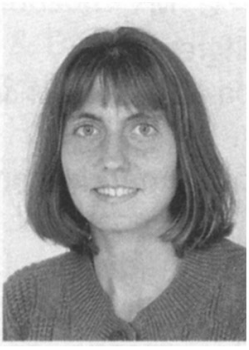

Gladys A. Ganiel

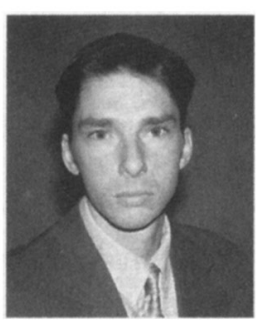

Aaron Z. Hale

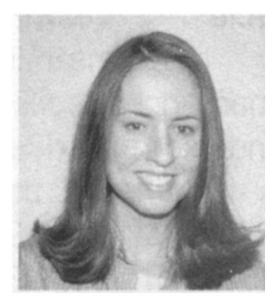

Jennifer L. Hildreth

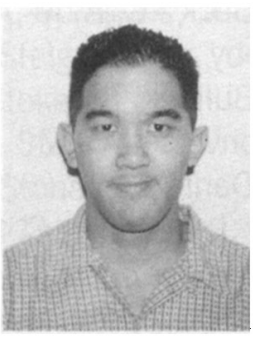

Daniel A. Weiss

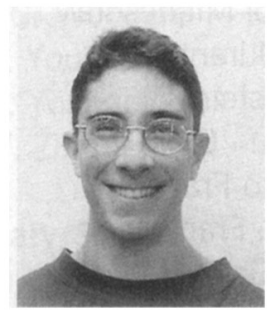

Neil G. Ruiz
Best Chapter Advisor Awards Awards of $\$ 1000$ go to faculty advisors who have served for at least three years and who have led their local chapters in an outstanding way.

Stephen Farnsworth, Mary Washington College

Eugene Garfield, Murray State University John C. Kuzenski, The Citadel James Wunsch, Creighton University

\section{Best Undergraduate Student Class Papers and Best Under- graduate Honors Thesis Awards $\$ 250$ for first place winners and $\$ 100$ each for runners-up.}

\section{Class Papers}

First Place: Amy Nelson, Moorhead State University, "Early Warning: A Collective Measure for the Prevention of Ethnic Conflict"

Runner-up: Mari K. Bonthius, St. Olaf College, "Has the Shooting Begun? A Test of the Culture War Theory on the Issue of Gay Rights"

Runner-up: Edwin W. Egee V, Mary Washington College, "Gaining Support through Casework: A Quantitative Study of Contractors and the Support They Give Their Representative"

\section{Honors Theses}

First Place: Jennifer L. Tate, Southwestern University, "A Living Past, a Dying Present: An Analysis of Nationalism, National Identity, and Ethnopolitics in the Conflict between Serbia and Bosnia-Herzegovina"

Runner-up: D. Michael Nardis, Georgetown University, "The Effect of Political Factors, Economic Conditions, and PAC Contributions on U.S. Senate Votes Concerning Chinese MFN Status in the Post-Tianamen Square Era"

Runner-up: Julia Tyurina, University of Arkansas, "Secular vs. Spiritual: The Development of the State in Medieval Political Thought" 


\section{Centennial (ampaign Contributors}

as of August 1, 1999

\section{Centennial Circle}

$(\$ 25,000+)$

Walter E. Beach - C, A, B, M

Doris Graber

Pendleton Herring

Huang Hsing Foundation \& Chun-tu

Hsueh

University of Louisville, in honor of

Aaron Wildavsky

Elinor \& Vincent Ostrom, in honor

of Alma Ostrom \& Leah Hopkins

Awan

Estate of Leo A. Shifrin

Frank J. Sorauf - C, M

1903 Circle
$(\$ 15,000+)$
Richard F. Fenno, Jr.
Norman H. Nie
Jack W. Peltason - C, A

\section{Circle}

$(\$ 10,000+)$

Ruth S. Jones \& Warren E. Miller -

C, M, A

Nannerl \& Robert O. Keohane - Br, B

Arend Lijphart

Dale Rogers Marshall

Pi Sigma Alpha

Founders Circle

$(\$ 5,000+)$

Lucius J. Barker

Robert H. Bates

Susan C. Bourque

David Brady

Roger H.\& Nancy Davidson - C, A,S

Jo Freeman - $W$

James L. Gibson

Betty Glad - P

John Mark Hansen

Martin O. Heisler - A

Jennifer $P$. Hochschild \& C. An-

thony Broh - C, B

Matthew Holden, Jr.
Gary C. Jacobson

Malcolm E. Jewell \& Sarah M.

Morehouse - C, M

Joyce K. Kallgren

Mary Fainsod Katzenstein \& Peter

Katzenstein

Martha Joynt Kumar, in honor of

Ann Devroy - P

Ruth P. Morgan - $P$

Norman J. Ornstein

Lucian W. Pye

J. Austin \& Nancy Ranney

Catherine E. Rudder - C, A, M, B,

$\mathrm{Br}, \mathrm{W}$

J. Merrill Shanks - M

Paul M. Sniderman

James $Q$. Wilson

Thomas R. Wolanin - S

Nancy H. Zingale

\section{Builders Circle}

$(\$ 2,500+)$

David Adamany

James $E$. Anderson

Anonymous - $\mathrm{B}$

William I. Bacchus

Michael A. Baer

Barbara B. Bardes

Paul Allen Beck - C, A, M

John F. Bibby

Charles S. Bullock, III

Philip E. Converse - C, M

William J. Daniels

Christopher J. Deering - C, S

Jorge I. Dominguez

Lawrence C. Dodd, in honor of the

Department of Political Science,

University of Minnesota

Robert F. Durant - V

Leon D. Epstein

Heinz Eulau - C, A

Luis Ricardo Fraga

Kathleen A. Frankovic

Virginia H Gray - C, A

Joel B. Grossman

Charles D. Hadley - C, A
Robert J-P. Hauck - C, A, M

Anne Hopkins

Robert Huckfeldt

$M$. Kent Jennings

Loch K. Johnson

Charles $O$. Jones - C, A, P

William J. Keefe

Rita Mae Kelly - B

Herbert P. Kitschelt

Allan Kornberg

Kay Lawson

Avery Leiserson

Burdett A. Loomis

Susan A. MacManus - C, A, B

David B. Magleby - S

Thomas E. Mann

Joel Margolis - S

David R. Mayhew

Daniel A. Mazmanian

Paula D. McClain

Cynthia McClintock

Lorraine M. McDonnell \& M.

Stephen Weatherford - $P$

Karen O'Connor

David Scott Palmer, in memory of

Eldon "Bud" Kenworthy

Leonard Parkinson

Michael B. Preston

George H. Quester

Beryl A. Radin

Randall Ripley

Alan Rosenthal

Kay Lehman Schlozman - C, B

Mildred A. Schwartz - C, M

Lee Sigelman

Howard J. Silver

Barbara Sinclair

Raphael J. Sonenshein

Harvey Starr

Dorothy McBride Stetson

Joseph Stewart, Jr.

Judith Hicks Stiehm - C, A

James A. Stimson - M

Clarence N. Stone

Edward Thompson, IIi, in honor of

Marguerite Ross Barnett \& Vincent

$\mathrm{C}=$ General Campaign Fund, $\mathrm{A}=$ Artinian Fund, $\mathrm{B}=$ Barnett Fund, $\mathrm{Br}=$ Bryce Fund, $\mathrm{M}=$ Miller Fund, $\mathrm{P}=$ Presidency Research Fund, $\mathrm{V}=$ Volcker Fund, $\mathrm{W}=$ Women \& Politics Fund 


\section{Celdrating the past by investing in the fiture}

J. Browne - B

James $A$. Thurber

David B. Truman

Sidney Verba

John C. Wahlke - C, A, P

Charls E. Walker

Kenneth N. Waltz

Paul J. Weber

Susan Welch

David Wilsford, in honor of Warren

Miller - C, M

\section{Second Century Society}

$(\$ 1,000+)$

Anthony Affigne - $B$

Herbert Alexander - A, M

Hayward R. Alker

Gabriel Almond

Patricia Artinian - A

Herbert B. Asher

Samuel H. Barnes - A, M

Jeffrey M. Berry

James W. Bjorkman

Christopher J. Bosso, in honor of

the Political Science faculty at the

University of Pittsburgh

Steven J. Brams

Michael A. Brintnall

Frances Burke

James MacGregor Burns

Lief $H$. Carter

Leonard Champney - A

Clarke E. Cochran

Thomas E. Cronin - C, P

Milton C. Cummings, Jr.

Robert A. Dahl

Jane $E$. Fountain, in honor of

Robert C. Wood - V

Linda Fowler

Jurg Martin Gabriel

Louis C. Gawthrop - A

George J. Graham, Jr. - A

Susan W. Hammond

Roderick P. Hart

C. Randall Henning

Mary A. Hepburn, in honor of
Sheilah Mann

Robert J. Huckshorn

William Hudson - A

Robert P. \& Dixie S. Huefner, in

honor of Edward Banfield

Karen M. Hult - P

Dennis Ippolito

Bruce W. Jentleson

Nolan E. Jones - B

David C. Leege - M

Gerhard Loewenberg

William Mishler, in honor of Allan

Kornberg - C, A, S

Janet M. Martin - $P$

John S. Odell

Kenneth T. Palmer

Desiree Pedescleaux - A

Nelson W. Polsby - A

Henry Pratt, in honor of David

Truman

David E. Price

Thomas F. Remington

Leroy N. Rieselbach - S

Donna E. Shalala - B

W. Phillips Shively - M

Roberta S. Sigel - C, B

Elliot E. Slotnick, in honor of Frank

J. Sorauf

Thomas A. Spragens, Jr.

Janet D. Steiger - S

Sybil L. Stokes

Gerald H. Stollman

Deborah Stone - C, B

Raymond Tatalovich - $P$

Charles E. Walcott - $P$

Raymond F. Wolfinger, in honor of

Warren E. Miller - M

Maurice C. Woodard

James V. Young

Robert L. Youngblood - M

Joseph F. Zimmerman

\section{Anniversary Society}

$(\$ 500+)$

Joel $D$. Aberbach, in honor of

Robert Lane
Martha Ackelsberg - B

Association of Chinese Political

Studies

Henry Bienen

Janet K. Boles - B, P

Ann O'Meara Bowman

Richard A. Brody - M

Emmett Buell - A

Bruce Bueno de Mequita

David A. Caputo

David Collier \& Ruth Berins Collier

M. Margaret Conway

Gary W. Cox

C. Jeremy Curtoys

Vincent \& Anne Davis, in honor of

Charles 0 . Jones

George C. Edwards, III - P

John B. Gates, in memory of

Richard Sinopoli

Harvey Glickman, in memory of $\mathrm{H}$.

Hubert Wilson

Kenneth \& Amanda Goldstein - M

Robert T. Golembiewski

Robert C. Grady - A

Melinda Gann Hall - A, B

William A. Hazleton \& Sandra Woy-

Hazleton

John R. Hibbing

Kim Quaile Hill - M

Harry N. Hirsch

John W. Holcombe, in memory of

George S. Blair

Patricia A. Hurley - M

W. Landis Jones

Peter Juviler - A

Miles Kahler

Samuel A. Kirkpatrick - M

Margaret Levi

Mark Lichbach

David L. Lowery

Naomi B. Lynn - B

L. Sandy Maisel, in honor of

Warren E. Miller - M

Lynn Mather - B

J. Donald Moon

Robert Montjoy

$\mathrm{C}=$ General Campaign Fund, $\mathrm{A}=$ Artinian Fund, $\mathrm{B}=$ Barnett Fund, $\mathrm{Br}=$ Bryce Fund, $\mathrm{M}=$ Miller Fund, $P=$ Presidency Research Fund, $V=$ Volcker Fund, $W=$ Women \& Politics Fund 


\section{Centennial (ampaign Contributors}

Robert J. Mundt

Richard W. Murray

Richard E. Neustadt - P

Pippa Norris - M

Joseph S. Nye, Jr.

Carole Pateman - A

Mark P. Petracca, in honor of Gina

\& Joseph Petracca

Gerald M. Pomper - A

Jewel L. Prestage - B

Robert D. Putnam

Donald L. Robinson

Bert A. Rockman - A

Lloyd I. \& Susanne Hoeber Rudolph

- C, B

Virginia Sapiro

Molly Lyndon Shanley - B

Robert Y. Shapiro - P

Beth Simmons

Harold Stanley - A

Randall Strahan

Norman C. Thomas - $P$

Jeffrey $K$. Tulis - $P$

Stephen L. Wasby - A, B, M, S - in

memory of Morris Udall

Marcia Lynn Whicker, in honor of

Malcolm Jewell - A

Frederick M. Wirt - B

Kenneth K. Wong

Diel S. Wright

David Vogel

\section{Campaign Friends}

$(\$ 250+)$

J. Theodore Anagnoson

Donald G. Balmer

John C. Berg - A

Gayle Binion

Mary Anne Borrelli - P

Randall L. Calvert

David T. Canon - S

Conference Group on Taiwan

Studies - Br

Constance Ewing Cook

Cary R. Covington - $P$

Russell Dalton - A

John G. Geer - M
Joyce Gelb - C, B

John W. Harbeson

Robert A. Heineman - A

Marjorie R. Hershey - A

Allen D. Hertzke

Raymond F. Hopkins

E. Terrence Jones - $M$

Mark P. Jones - M

Marion R. Just - A

William R. Keech - A

Scott Keeter - A

Frank Kessler - $P$

John Kincaid

Samuel Krislov \& Judith Gillespie -

A

David A. Lake

David D. Laitin

Logan A. Lee

Andrew S. McFarland - A

Eugene Meehan - A

Bruce Miroff - A

New England Political Science

Association - A

Richard G. Niemi

Susan S. Northcutt

James L. O'Sullivan - Br

Don Racheter

David M. Rayside

Richard \& Rosemary Rose - A

Herbert J. Rubin - A

Arlene W. Saxonhouse - M

W. Rand Smith

Carol M. Swain - C, A, B

Santa \& Michael Traugott - $M$

Clyde Wilcox

Shirley Anne Warshaw - P

M. Crawford Young

\section{Campaign Associates}

(\$100-\$249)

Paul. R. Abramson - M

Shariff Ahmad - S

Gar Alperovitz

Asher Arian - A

Harold F. Bass, Jr. - C, A

David J. Bellshaw - M

David R. Berman
Nancy Bermeo - B

Janet Box-Steffensmeier - C, A, B, $M, S$

Evelyn S. Brewster - M

Thomas M. Callaghy

Colin Campbell - A

Jeffrey E. Cohen - P

William J. Crotty

I.M. Destler - A

Donna Robinson Divine

Michael W. Doyle \& Amy Gutmann

Malcolm Feeley - A

Paul Ferber

Jane Flax - B

John P. \& Lorraine W. Frank - M

Michael Genovese - $P$

Emily R. Gill - B

Girls Ranch, Inc. of Arizona - M

Nicole A. Gordon - M

Joanne Gowa

Fred I. Greenstein - C, P

Joseph M. Grieco

Richard and Cheryl Herrera - M

Paul S. Herrnson - A

Kenneth Hoover, in memory of

Edward Artinian - A

Serge Hurtig - C, M

Jim Moss \& Company - M

Mary Donovan Jones - M

Ronald Kahn

Ellis Katz

Christopher Kelaher - A

Donald F. Kettl - A

John J. Kirlin

Michael G. Krukones

Susan S. Lederman - A

Michael H. Levin - S

Michael Lienesch

Roderick MacFarquhar

Harvey C. Mansfield, Jr. - A

Scott L. McLean - A

Wilson Carey McWilliams - A

Gabriel Miller - A

Harris N. Miller - S

Kristen Monroe

Charles C. Moskos, Jr.

Elizabeth F. Moulds

$$
\begin{aligned}
& \mathrm{C}=\text { General Campaign Fund, } \mathrm{A}=\text { Artinian Fund, } \mathrm{B}=\text { Barnett Fund, } \mathrm{Br}=\text { Bryce Fund, } \mathrm{M}=\text { Miller Fund, } \\
& P=\text { Presidency Research Fund, } V=\text { Volcker Fund, } W=\text { Women \& Politics Fund }
\end{aligned}
$$




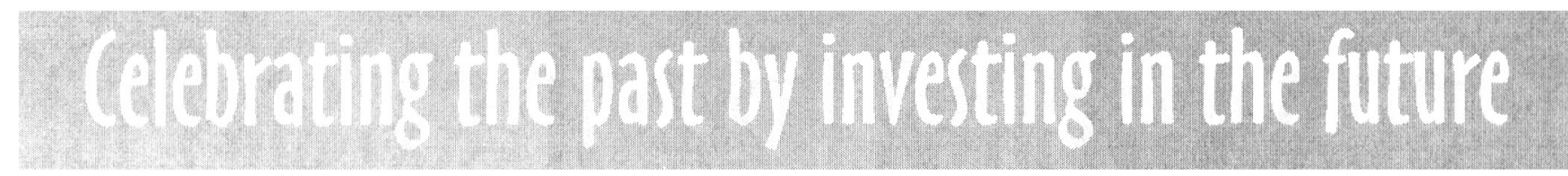

Susanne D. Mueller - B

Carol Nechemias

David Newman - A

Joseph L. Nogee

Helmut Norpoth - A, M

Marian Lief Palley - B

Samuel C. Patterson - A

Thomas E. Patterson - A

William D. Pederson

Jack Pfister - M

John C. Pierce - M

Dianne Pinderhughes - B

Richard Pious - $P$

Portal Pine Creek Canyon

Homeowners Assocation Unit III -M

Jorgen Rasmussen - A

Representation \& Electoral Systems

Organized Section

Margaret A. Robinson - M

Nancy L. Rosenblum

Frances M. Rosenbluth

Margaret Ann Rosenthal - A

Anne L. Schneider - B

Byron E. Shafer - A

Kenneth A. Shepsle - M

Steven A. Shull - $P$

Sheldon W. Simon - M

Peter N. Skerry

Carolyn M. Shrewsbury - B

Robert J. Spitzer - A

Phillippa Strum - B

Kathleen Tapport - B

Leslie Paul Thiele - A

Emma S. Thomas - $M$

Joan Hulse Thompson - B

J. Ann Tickner - B

Kenneth D. Wald - A

Herbert Waltzer - A

Robert E. Ward

Stephen White - A

Donald \& Margaret Wickler - M

Clyde Wilcox - A

Kay Williams - M

Robert C. Wood - A

Aristide R. Zolberg - B

Alan Zuckerman - A

\section{Contributors}

(\$0-\$99)

Karen J. Alter

David M. Barrett - P

Donald C. Baumer

Seyla Benhabib

Frederic $A$. Bergerson

Thomas E. Borcherding

Evelyn Z. Brokin

John E. Brown - M

Frances Burke - A

Sara G. Carpenter - M

Brenda W. Carter - A

Allan J. Cigler - A

Caleb M. Clark - A

Patrick Coby

Michael J. Coppedge

Alex N. Dragnich - A

Pat M. Dunham - A

Valerie Earle - $M$

Cynthia Enloe - A

James W. Fesler - A

Timothy Fuller

Peggy Glick - M

Naomi \& David Goodell

Lawrence Graham - A

Martin Gruberg - B

Glen Halva-Neubauer

Roger Hamburg - A

Jonathan Hartlyn

Barbara J. Hayler - B

Alice L. Hearst

Charles F. Hermann

Frederick M. Herrmann - M

Dean R. Hewitt

Erik P. Hoffmann - A

Susan E. Howell

Malcolm E. Jewell - A

Charlotte Joseph

Junko Kato

Herbert Kaufman - A

Richard C. Kearney - A

Anthony King - A

Henry Krisch - A

Lan Thuc Le

Jerome S. Legge, Jr. - A

Lance Leloup
Jonathan Lemco - A

Elaine S. Levine - A

Jeremy R.T. Lewis - A

Robert C. Lieberman - A

Charles H. Lipson

Lyceum Books, Inc. - A

Elaine P. Maimon - $M$

Michael Margolis - A

Suzanne M. Marilley - C, B

Mark G. Mazzie - M

Margaret \& Ken McBeth - M

Clyde D. Mckee, Jr. - A

Richard L. Merritt - A

David R. Morgan - A

Stuart S. Nagel - A

Univ of North Carolina, Chapel Hill, Dept. of Political Science - M

Anne M. Parsons

Mark A. Peterson - A

Edward B. Portis - A

Wendy M. Rahn - A

Ronald B. Rapoport - S

Paul Rejai - A

Ross R. Rice - M

David B. Robertson - B

Bernard H. Ross - A

Blair Ruble

Wilma Rule - B

Cynthia \& Donald Ryan

Laura J. Scalia - A

Beth S. Schapiro

Jean Reith Schroedel - B

L. Earl Shaw, Jr. - A

Susan J. Shearon - M

Henry B. Sirgo - A

Theda Skocpol - B

Rosemary Glass Spalding - M

Robert J. Spitzer - A

Kathryn Dunn Tenpas

Charles B. Turpin

Henry Valen - M

Diane E. Wall - B

Retha W. Warnicke - M

Marvin G. Weinbaum - A

Gregory W. White

Janet L. Womack

Iris Marion Young

$\mathrm{C}=$ General Campaign Fund, $\mathrm{A}=$ Artinian Fund, $\mathrm{B}=$ Barnett Fund, $\mathrm{Br}=$ Bryce Fund, $\mathrm{M}=$ Miller Fund, $P=$ Presidency Research Fund, $V=$ Volcker Fund, $W=$ Women \& Politics Fund 


\section{余 Annual Reviews}

\section{Special Offer! Purchase Volume 2 at a 20\% discount AND receive Volume 1 FREE OF CHARGE}

Volume 2

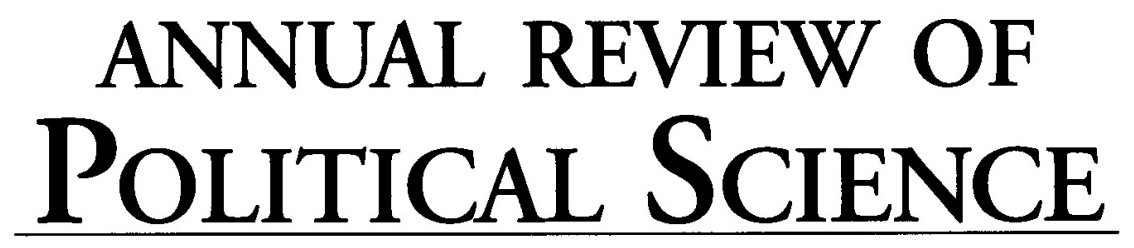

June 1999

EDITOR: Nelson W. Polsby, University of California at Berkeley

Editorial and Planning Committee Members:

David W. Brady, Stanford University • Bruce Bueno de Mesquita, The Hoover Institution •

Jean Bethke Elshtain, University of Chicago $\bullet$ Donald P. Green, Yale University •

Robert L. Jervis, Columbia University • Margaret Levi, University of Washington •

Nelson W. Polsby, University of California at Berkeley•

Sidney G.Tarrow, Cornell University • Alan J.Ware, Oxford University

\section{What your distinguished colleagues are saying...}

"With the flood of research articles increasing yearly,

political science has long needed an annual review." - Christopher H. Achen

Professor, Political Science, Research Scientist, ISR, University of Michigan

"I expect that our colleagues throughout the world will find the Annual Review of Political Science an indispensable source of knowledge."

- Heinz Iulau, Stanford University

"This is a really impressive beginning for the series, one that sets a standard so high that successive volumes will be challenged to reach."

- John Ferejohn, Professor of Political Science, Stanford University

\section{Subscribe Today!}

Order your personal subscription of Annual Reviews OR please forward this information to your librarian to add this volume to your institution's collection. Place a standing order and receive a $10 \%$ discount each year!

\section{Online Access When You Need It!}

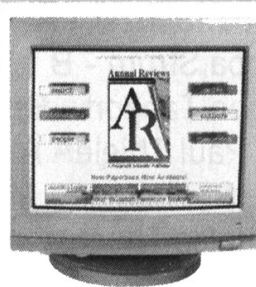

Annual Reviews Online at www.AnnualReviews.org Current print subscribers to Annual Reviews receive free online access to each series that they subscribe to, as well as access to back volumes as they become available.

Discount Price: \$48 USA/\$52 Foreign - Regular Price: Individuals \$60 USA/\$65 Foreign • Institutions \$120 USA/\$130 Foreign • Page Count: 565 Place your orders directly with Annual Reviews. PRIORITY ORDER CODE: JA-APSA29. Please mention this code when placing orders.

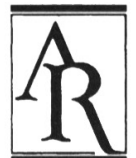

ANNUAL REVIEWS

a nonprofit scientific publisher

4139 El Camino Way

P.O. Box 10139

Palo Alto, CA 94303-0139 USA

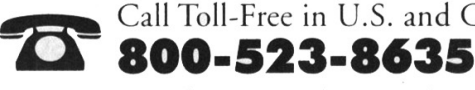

Call from anywhere in the world 650-493-4400 Ext. 1
FAX from anywhere in the world 650-424-0910

World Wide Web www.AnnualReviews.org E-mail service@annurev.org 